\title{
On a fundamental result in van der Corput's method of estimating exponential sums
}

\author{
by \\ HoNG-QUAN LiU (Harbin)
}

1. Introduction. In the early 1920 s, van der Corput developed a powerful method to estimate exponential sums of the following type (cf. [18], Chap. 4):

$$
S=\sum_{a \leq m \leq b} e(f(m))
$$

where $1 \leq a \leq b \leq 2 a, m$ runs through integers, $e(\xi)=\exp (2 \pi i \xi)$ for real $\xi$, and $f(x)$ is a real function such that at least the first derivative $f^{\prime}(x)$ exists for $x \in[a, b]$. Apart from the well-known Weyl inequality, another basic procedure of the method is to transform the estimate of the sum $S$ to the estimate of another exponential sum. Precisely speaking, if the second derivative $f^{\prime \prime}(x)$ is continuous and does not change sign on $[a, b]$, then

$$
S=\lambda \sum_{\alpha<v<\beta}\left|f^{\prime \prime}\left(x_{v}\right)\right|^{-1 / 2} e\left(f\left(x_{v}\right)-v x_{v}+1 / 8\right)+E,
$$

where $\lambda=1$ or $-i$ according as either $f^{\prime \prime}>0$ or $f^{\prime \prime}<0, x_{v}$ is the solution of $f^{\prime}(x)=v, \alpha \leq v \leq \beta, \alpha=\min \left(f^{\prime}(a), f^{\prime}(b)\right), \beta=\max \left(f^{\prime}(a), f^{\prime}(b)\right)$, and $E$ is the error term. The problem left to be settled is how we can get a better estimate for $E$. If $f^{(3)}(x)$ is continuous on $[a, b], f^{\prime}(x)$ is decreasing on $[a, b]$, and

$$
\lambda_{2} \leq\left|f^{\prime \prime}(x)\right| \leq A \lambda_{2}, \quad\left|f^{(3)}(x)\right| \leq A \lambda_{3}, \quad \lambda_{2}>0, \lambda_{3}>0,
$$

and $A$ is a positive constant, then van der Corput showed (cf. [18], Theorem 4.9) that

$$
E=O\left(\lambda_{2}^{-1 / 2}\right)+O\left(\log \left(2+(b-a) \lambda_{2}\right)\right)+O\left((b-a) \lambda_{2}^{1 / 5} \lambda_{3}^{1 / 5}\right) .
$$

1991 Mathematics Subject Classification: 11L26, 11L30.

Research supported by Harbin Institute of Technology and the National Science Foundation of China. 
Assuming the additional condition that $f^{(4)}(x)$ is continuous on $[a, b]$ and satisfies

$$
\left|f^{(4)}(x)\right| \leq A \lambda_{4}, \quad \lambda_{4}>0, \lambda_{3}^{2}=\lambda_{2} \lambda_{4},
$$

Phillips [17] showed that

$$
E=O\left(\lambda_{2}^{-1 / 2}\right)+O\left(\log \left(2+(b-a) \lambda_{2}\right)\right)+O\left((b-a) \lambda_{3}^{1 / 3}\right) .
$$

Although this estimate suffices to establish the theory of exponent pairs, it is not useful for estimating several multiple exponential sums. Therefore, Heath-Brown [3] got an important improvement by means of complex analysis. Assume that $f(z)$ is analytic in a domain $R$ containing the interval $[a, b], R^{\prime}=\{z \mid a z \in R\}$ is an open convex set, and $\left|f^{\prime \prime}(z)\right| \leq M$ holds for $z \in R$. Moreover, for a real number $x \in R, f^{\prime \prime}(x) \leq-A M$, where $A>0$ is a positive constant. Then Lemma 6 of [3] gives

$$
E=O\left(M^{-1 / 2}\right)+O(\log (2+(b-a) M)) .
$$

It is noteworthy that Kolesnik already stated essentially the same result as Lemma 2 of [5] in 1982, but gave no detailed proof. Heath-Brown's estimate for the error term $E$ is of course good enough, and it has been used in the investigation of many problems (cf. [3], [6], [7], [13]-[15]). However, it does not suffice in the estimation of many multiple exponential sums, especially those coming from multiple divisor problems (cf. [2], [4], [8]-[12], [19], [20]). Assuming that $f(x)$ is an algebraic function for $x \in[a, b]$, and

$$
R^{-1} \leq\left|f^{\prime \prime}(x)\right| \leq A R^{-1}, \quad\left|f^{(3)}(x)\right| \leq A(R U)^{-1}, \quad U \geq 1,
$$

Min [16] got a stronger estimate for $E$. Theorem 2.2 of [16] gives

$$
\begin{aligned}
E= & E_{1}+E_{2}+O\left(\log \left(2+(b-a) R^{-1}\right)\right)+O\left((b-a+R) U^{-1}\right) \\
& +O\left(\min \left(R^{1 / 2}, \max (1 /\langle\alpha\rangle, 1 /\langle\beta\rangle)\right)\right),
\end{aligned}
$$

where

$$
\begin{aligned}
& E_{1}=\lambda b_{\alpha}\left|f^{\prime \prime}\left(x_{\alpha}\right)\right|^{-1 / 2} e\left(f\left(x_{\alpha}\right)-\alpha x_{\alpha}+1 / 8\right), \\
& E_{2}=\lambda b_{\beta}\left|f^{\prime \prime}\left(x_{\beta}\right)\right|^{-1 / 2} e\left(f\left(x_{\beta}\right)-\beta x_{\beta}+1 / 8\right),
\end{aligned}
$$

$b_{\alpha}=1 / 2$ if $\alpha$ is an integer, otherwise $b_{\alpha}=0$, and $b_{\beta}$ is defined similarly, $\langle\alpha\rangle=\beta-\alpha$ if $\alpha$ is an integer, and otherwise $\langle\alpha\rangle=\|\alpha\|=\min _{n \in \mathbb{Z}}|n-\alpha|$, and $\langle\beta\rangle$ is defined similarly. Before Jia's work [4] and the author's work [8], Min's result was neither known abroad nor used domestically. In fact Jia [4] only used a consequence of Min's result, that is (note that $E_{1}=O\left(b_{\alpha} R^{1 / 2}\right)$ ),

$$
\begin{aligned}
E= & O\left(\min \left(R^{1 / 2}, 1 /\|\alpha\|\right)\right)+O\left(\min \left(R^{1 / 2}, 1 /\|\beta\|\right)\right) \\
& +O\left(\log \left(2+(b-a) R^{-1}\right)\right)+O\left((b-a+R) U^{-1}\right) .
\end{aligned}
$$

Min's result plays a decisive role in recent deeper investigations of certain exponential sums (cf. [2], [4], [8]-[12], [19], [20]). However, the statement of Min's result depends on the deep notion of "algebraic function" (cf. Chapter 
8 of [1] for a precise definition), which makes its proof obscure (it will be clear later where this deep notion is appealed to). The dependence on such a deep notion is also not in consistence with van der Corput's original method, which depends only on properties of derivative functions. Moreover, Min's method does not even yield a direct proof for the simple case where $f(x)$ is a monomial. In this paper, we shall show that if the derivatives of $f(x)$ satisfy suitable conditions, then Min's estimate for the error term $E$ of van der Corput's formula still holds true. Let $f^{(k)}(x)$ denote the derivative of $f$ of order $k, 2 \leq k \leq 5$.

THEOREM 1. Let $f(x)$ be a real function such that $f^{(5)}(x)$ is a continuous function for $x \in[a, b], C_{k}(1 \leq k \leq 6)$ be certain positive constants,

$$
\begin{gathered}
C_{1} R^{-1} \leq\left|f^{(2)}(x)\right| \leq C_{2} R^{-1}, \quad\left|\beta_{k}(x)\right| \leq C_{k} U^{2-k}, \quad U \geq 1,3 \leq k \leq 5, \\
\beta_{k}(x)=f^{(k)}(x) / f^{(2)}(x) .
\end{gathered}
$$

Assume that $\left|3 \beta_{4}(x)-5 \beta_{3}^{2}(x)\right| \geq C_{6} U^{-2}$ for all $x \in[a, b]$. Then

$$
\begin{gathered}
\sum_{a \leq m \leq b} e(f(m))=\lambda \sum_{\alpha<v<\beta}\left|f^{\prime \prime}\left(x_{v}\right)\right|^{-1 / 2} e\left(f\left(x_{v}\right)-v x_{v}+1 / 8\right)+E \\
E=E_{1}+E_{2}+O\left(\log \left(2+(b-a) R^{-1}\right)\right)+O\left((b-a+R) U^{-1}\right) \\
+O\left(\min \left(R^{1 / 2}, \max (1 /\langle\alpha\rangle, 1 /\langle\beta\rangle)\right)\right)
\end{gathered}
$$

where $E_{1}, E_{2}$, and all other symbols are defined as before.

Applying Theorem 1, we can prove the following

Theorem 2. If $f(x)=B x^{\delta}, B \delta \neq 0, \delta \neq 1,2$, then (1) holds with $R=\left(|B| a^{\delta-2}\right)^{-1}, U=a$.

Exponential sums weighted by the factor $m^{-1 / 2}$ are of particular interest in case $f(x)$ is a monomial. Using Theorem 1 , we can easily get

TheOREM 3. If $f(x)=B x^{\delta}, \delta B>0, \delta \neq 1,2$, then

$$
\begin{aligned}
& \sum_{a \leq m \leq b} m^{-1 / 2} e(f(m)) \\
& =C \lambda \sum_{\alpha<v<\beta} v^{-1 / 2} e(g(v))+E_{1}+E_{2}+O\left(a^{-1 / 2} \log \left(2+(b-a) R^{-1}\right)\right) \\
& \quad+O\left((b-a+R) a^{-3 / 2}\right)+O\left(a^{-1 / 2} \min \left(R^{1 / 2}, \max (1 /\langle\alpha\rangle, 1 /\langle\beta\rangle)\right)\right),
\end{aligned}
$$

where $C=(|\delta-1|)^{-1 / 2}, \lambda=1$ or $-i$ according as $\delta>1$ or $\delta<1$, $\alpha=\min \left(f^{\prime}(a), f^{\prime}(b)\right), \beta=\max \left(f^{\prime}(a), f^{\prime}(b)\right), g(v)=(\delta B)^{\tau} v^{\eta}\left(\delta^{-1}-1\right), \tau=$ $1 /(1-\delta), \eta=\delta /(\delta-1), E_{1}=C \lambda \alpha^{-1 / 2} b_{\alpha} e(g(\alpha)), E_{2}=C \lambda \beta^{-1 / 2} b_{\beta} e(g(\beta))$, the notations $b_{\alpha}, b_{\beta},\langle\alpha\rangle$ and $\langle\beta\rangle$ are defined as before, and $R=\left(|B| a^{\delta-2}\right)^{-1}$. 
Theorems 2 and 3 show the validity of all the results in the literature derived from Min's result, without depending on the notion of "algebraic function".

\section{Lemmas}

LEMMA 1. Let $f(x)$ be a real function on $[a, b]$ such that $f^{\prime}(x)$ is continuous and decreasing, $f^{\prime}(a)=\beta, f^{\prime}(b)=\alpha$, and $g(x)$ is a positive decreasing function defined on the same interval with $g^{\prime}(x)$ continuous and $\left|g^{\prime}(x)\right|$ decreasing. Then for every positive constant $\varphi$ smaller than 1 we have

$$
\begin{aligned}
\sum_{a \leq n \leq b} g(n) e(f(n))= & \sum_{\alpha-\varphi<v<\beta+\varphi} \int_{a}^{b} g(x) e(f(x)-v x) d x \\
& +O(g(a) \log (\beta-\alpha+2))+O\left(\left|g^{\prime}(a)\right|\right) .
\end{aligned}
$$

Proof. This is Lemma 4.10 of [18].

LEMMA 2. If $F(x)$ and $G(x)$ are real functions on $[a, b]$ such that $G(x) / F^{\prime}(x)$ is monotonic, and $\left|G(x) / F^{\prime}(x)\right| \leq M$ for all $x \in[a, b]$, then

$$
\int_{a}^{b} G(x) e(F(x)) d x=O(M) .
$$

Proof. This is Lemma 4.3 of [18].

LEMMA 3. If $F(x)$ is a real function such that $F^{\prime \prime}(x)$ is continuous and $\left|F^{\prime \prime}(x)\right| \geq r>0$ for all $x \in[a, b]$, and $G(x)$ is a real function such that $|G(x)| \leq M$ and $G(x)$ is monotonic on $[a, b]$, then

$$
\int_{a}^{b} G(x) e(F(x)) d x=O\left(M r^{-1 / 2}\right) .
$$

Proof. This is Lemma 4.5 of [18].

LEMma 4. Let $M>N>0$. Then

$$
\begin{aligned}
\int_{N}^{M} e\left(x^{2}\right) d x= & (1+i) \int_{N}^{M} \exp \left(-4 \pi x^{2}\right) d x \\
& +O(\min (N, 1 / N))+O\left(\frac{1-\exp \left(-4 \pi M^{2}\right)}{M}\right),
\end{aligned}
$$

(ii) $\quad \int_{N}^{\infty} e\left(x^{2}\right) d x=O(\min (1,1 / N))$,

(iii) $\int_{0}^{\infty} e\left(x^{2}\right) d x=\frac{1}{4}(1+i)$, 
(iv)

$$
\int_{N}^{\infty} e(x) x^{-1 / 2} d x=O\left(\min \left(1, N^{-1 / 2}\right)\right),
$$

(v)

$$
\int_{0}^{\infty} e(x) x^{-1 / 2} d x=\frac{1}{2}(1+i) .
$$

Proof. By the residue theorem we get

$$
\int_{L} e\left(z^{2}\right) d z=0,
$$

where $L$ is the closed path consisting of the four sides of the quadrangle of the complex plane with vertices $(N, 0),(M, 0),(M, M)$ and $(N, N)$. Thus

$$
\int_{N}^{M} e\left(x^{2}\right) d x=R_{1}+R_{2}+R_{3},
$$

where $R_{1}, R_{2}$ and $R_{3}$ are the integrals over the sides from $(M, M)$ to $(M, 0)$, $(N, N)$ to $(M, M)$, and $(N, 0)$ to $(N, N)$ respectively. We have

$$
\begin{aligned}
\left|R_{1}\right| & =\left|\int_{0}^{M} \exp \left(2 \pi i(M+i y)^{2}\right) d y\right|=O\left(\int_{0}^{M} \exp (-4 \pi M y) d y\right) \\
& =O\left(\frac{1-\exp \left(-4 \pi M^{2}\right)}{M}\right) \\
R_{2} & =\int_{N}^{M} \exp \left(2 \pi i(t+t i)^{2}\right)(1+i) d t=(1+i) \int_{N}^{M} \exp \left(-4 \pi t^{2}\right) d t \\
\left|R_{3}\right| & =\left|\int_{0}^{N} \exp \left(2 \pi i(N+i y)^{2}\right) d y\right|=O\left(\int_{0}^{N} \exp (-4 N y) d y\right) \\
& =O\left(\frac{1-\exp \left(-4 \pi N^{2}\right)}{N}\right) .
\end{aligned}
$$

Now $R_{3}=O(1 / N)$ is obvious. For sufficiently small $N, \exp \left(-4 \pi N^{2}\right)=$ $1+O\left(N^{2}\right)$. This shows that $R_{3}=O(\min (N, 1 / N))$, and (i) follows. (ii) follows from (i) by letting $M \rightarrow \infty$ and noting that

$$
\begin{aligned}
& \int_{N}^{\infty} \exp \left(-4 \pi x^{2}\right) d x<\int_{0}^{\infty} \exp \left(-4 \pi x^{2}\right) d x=O(1), \\
& \int_{N}^{\infty} \exp \left(-4 \pi x^{2}\right) d x<\int_{N}^{\infty} \exp (-4 \pi N x) d x=O(1 / N) .
\end{aligned}
$$


Letting $M \rightarrow \infty$ and $N \rightarrow 0$, from (i) we get

$$
\int_{0}^{\infty} e\left(x^{2}\right) d x=(1+i) \int_{0}^{\infty} \exp \left(-4 \pi x^{2}\right) d x:=(1+i) I, \quad \text { say. }
$$

It suffices for us to calculate the integral $I$. We have

$$
I^{2}=\int_{0}^{\infty} \int_{0}^{\infty} \exp \left(-4 \pi\left(x^{2}+y^{2}\right)\right) d x d y
$$

Let $x=r \cos \theta, y=r \sin \theta, 0 \leq r<\infty$, and $0 \leq \theta \leq \pi / 2$. This changes the double $(x, y)$ integral into a double $(r, \theta)$ integral, and we get

$$
I^{2}=\int_{0}^{\infty} \exp \left(-4 \pi r^{2}\right) r d r \int_{0}^{\pi / 2} d \theta=1 / 16
$$

which proves (iii). Letting $x=u^{2}$ in the integrals of (iv) and (v), it is easy to see that the conclusions follow from (ii) and (iii) respectively.

3. Proof of Theorem 1. As $f^{\prime \prime}(x)$ does not change sign on $[a, b]$, we can suppose without losing generality that $f^{\prime \prime}(x)<0$ for all $x \in[a, b]$. By Lemma 1 we have

(2) $\sum_{a \leq n \leq b} e(f(n))=\sum_{\alpha-1 / 2<v<\beta+1 / 2} \int_{a}^{b} e(f(x)-v x) d x+O(\log (\beta-\alpha+2))$.

Let $n_{v}$ be the unique number satisfying $f^{\prime}\left(n_{v}\right)=v$, where $\alpha \leq v \leq \beta$. Then $n_{v} \in[a, b]$. Assume that $n_{v} \neq b$. Let $\xi=b-n_{v}$. We have

$$
\begin{aligned}
\int_{n_{v}}^{b} e(f(x)-v x) d x & =\int_{0}^{\xi} e\left(f\left(t+n_{v}\right)-v\left(t+n_{v}\right)\right) d t \\
& =e\left(f\left(n_{v}\right)-v n_{v}\right) \int_{0}^{\xi} e(A(t)) d t,
\end{aligned}
$$

where $A(t)=f\left(t+n_{v}\right)-f\left(n_{v}\right)-v t$. Let $\varepsilon$ be a sufficiently small given positive constant depending on the constants $C_{k}(2 \leq k \leq 6)$. If $t \geq \varepsilon U$, then

$$
A^{\prime}(t)=f^{\prime}\left(t+n_{v}\right)-f^{\prime}\left(n_{v}\right)=t f^{\prime \prime}\left(t_{1}+n_{v}\right) \leq-\varepsilon U R^{-1},
$$

where $t_{1} \in[0, t]$. Thus by Lemma 2 we get

$$
\int_{c}^{\xi} e(A(t)) d t=O\left(R U^{-1}\right)
$$


where $c=\min (\xi, \varepsilon U)$. The leading term in the Taylor expansion of $A(t)$ is $\frac{1}{2} f^{\prime \prime}\left(n_{v}\right) t^{2}$, thus we may approximate the integral

$$
J=\int_{0}^{c} e(-A(t)) d t
$$

by

$$
\int_{0}^{\infty} e\left(-\frac{1}{2} f^{\prime \prime}\left(n_{v}\right) t^{2}\right) d t=\left(-\frac{1}{2} f^{\prime \prime}\left(n_{v}\right)\right)^{-1 / 2} \frac{1}{2} \int_{0}^{\infty} e(x) x^{-1 / 2} d x .
$$

Let $-A(t)=u$. We have

$$
J=\int_{0}^{d} e(u)\left(-A^{\prime}(t(u))\right)^{-1} d u
$$

where $d=-A(c)>0$. Let $Y=\left(-\frac{1}{2} f^{\prime \prime}\left(n_{v}\right)\right)^{-1 / 2}$. We have

$$
J-\frac{1}{2} Y \int_{0}^{\infty} e(x) x^{-1 / 2} d x=\int_{0}^{d} e(u) F(u) d u-\frac{1}{2} Y \int_{d}^{\infty} e(u) u^{-1 / 2} d u
$$

where

$$
F(u)=-\frac{1}{A^{\prime}(t(u))}-\frac{1}{2} Y u^{-1 / 2}
$$

$A^{\prime}(\cdot)$ is the derivative of $A(\cdot)$, and $t(u)$ is the solution of $A(t)=-u$. We have

$$
F^{\prime}(u)=\frac{-A^{\prime \prime}(t(u))}{\left(A^{\prime}(t(u))\right)^{3}}+\frac{1}{4} Y u^{-3 / 2} .
$$

If there is $u \in(0, d]$ such that $F^{\prime}(u)=0$, then there is $t \in(0, c]$ such that

(6) $f^{\prime \prime}(t+n)\left(f^{\prime}(t+n)-f^{\prime}(n)\right)^{-3}=\frac{1}{4} Y(-f(t+n)+f(n)+v t)^{-3 / 2}$,

where we write $n=n_{v}$ for simplicity. We will deduce a contradiction from

(6) under the assumptions of Theorem 1. Taking Taylor expansions we get

$$
\begin{aligned}
f^{\prime \prime}(t+n) & =f^{(2)}(n)+t f^{(3)}(n)+\frac{1}{2} t^{2} f^{(4)}(n)+\frac{1}{6} t^{3} f^{(5)}\left(N_{1}\right), \\
f^{\prime}(t+n)-f^{\prime}(n) & =t f^{(2)}(n)+\frac{1}{2} t^{2} f^{(3)}(n)+\frac{1}{6} t^{3} f^{(4)}(n)+\frac{1}{24} t^{4} f^{(5)}\left(N_{2}\right), \\
f(t+n)-f(n)-v t & =\frac{1}{2} f^{(2)}(n) t^{2}+\frac{1}{6} f^{(3)}(n) t^{3}+\frac{1}{24} f^{(4)}(n) t^{4}+\frac{1}{120} f^{(5)}\left(N_{3}\right) t^{5},
\end{aligned}
$$

where $N_{1}, N_{2}$ and $N_{3}$ are suitable numbers in $[n, n+t]$. Thus from (6) and the assumptions of Theorem 1, we get

$$
\begin{gathered}
F_{1}(t)\left(F_{3}(t)\right)^{3 / 2}=\left(F_{2}(t)\right)^{3}, \\
F_{1}(t)=1+t \beta_{3}+\frac{1}{2} t^{2} \beta_{4}+c_{1} \theta_{1} t^{3} U^{-3}, \\
F_{2}(t)=1+X_{1}, \quad X_{1}=\frac{1}{2} t \beta_{3}+\frac{1}{6} t^{2} \beta_{4}+c_{2} \theta_{2} t^{3} U^{-3}, \\
F_{3}(t)=1+X_{2}, \quad X_{2}=\frac{1}{3} t \beta_{3}+\frac{1}{12} t^{2} \beta_{4}+c_{3} \theta_{3}(t / U)^{3},
\end{gathered}
$$


where $\beta_{k}=\beta_{k}(n), c_{i}$ is a suitable positive constant, and $\theta_{i}=\theta_{i}(t) \in[0,1]$. As $\left|\beta_{k}\right| \leq C_{k} U^{-k+2}, t \leq \varepsilon U$, we have $X_{2}=O(t / U)=O(\varepsilon)$, and

$$
\begin{aligned}
\left(F_{2}(t)\right)^{3} & =\left(1+X_{1}\right)^{3}=1+3 X_{1}+3 X_{1}^{2}+X_{1}^{3} \\
& =1+\frac{3}{2} t \beta_{3}+t^{2}\left(\frac{3}{4} \beta_{3}^{2}+\frac{1}{2} \beta_{4}\right)+c_{4} \theta_{4}(t / U)^{3}, \\
\left(F_{3}(t)\right)^{3 / 2} & =\left(1+X_{2}\right)^{3 / 2}=1+\frac{3}{2} X_{2}+\frac{3}{8} X_{2}^{2}+c_{5} \theta_{5}(t / U)^{3} \\
& =1+\frac{1}{2} t \beta_{3}+t^{2}\left(\frac{1}{8} \beta_{4}+\frac{1}{24} \beta_{3}^{2}\right)+c_{6} \theta_{6}(t / U)^{3}, \\
F_{1}(t)\left(F_{3}(t)\right)^{3 / 2} & =1+\frac{3}{2} t \beta_{3}+t^{2}\left(\frac{5}{8} \beta_{4}+\frac{13}{24} \beta_{3}^{2}\right)+c_{7} \theta_{7}(t / U)^{3} .
\end{aligned}
$$

Thus from (7) we get

$$
\frac{5}{24} \beta_{3}^{2}-\frac{1}{8} \beta_{4}+c_{8} \theta_{8} t U^{-3}=0,
$$

which is impossible, for we have $\left|3 \beta_{4}-5 \beta_{3}^{2}\right| \geq C_{6} U^{-2}$ and $t \leq \varepsilon U$. This shows that $F^{\prime}(u) \neq 0$ for $u \in(0, d]$. As $F^{\prime}(u)$ is a continuous function for $u \in(0, d]$, it follows that $F^{\prime}(u)$ does not change sign on $(0, d]$, which implies that $F(u)$ is monotonic on $(0, d]$. For $u \in(0, d]$, from

$$
u=-A(t)=-(f(n+t)-f(n)-v t)=-\frac{1}{2} f^{\prime \prime}(n) t^{2}(1+O(t / U))
$$

we get

$$
t=t(u)=Y u^{1 / 2}(1+O(t / U)) .
$$

Thus

$$
\begin{aligned}
A^{\prime}(t(u)) & =f^{\prime}(n+t)-f^{\prime}(n)=f^{\prime \prime}(n) t\left(1+O\left(t U^{-1}\right)\right) \\
& =-2 Y^{-1} u^{1 / 2}\left(1+O\left(t U^{-1}\right)\right), \\
F(u) & =-\frac{1}{A^{\prime}(t(u))}-\frac{1}{2} Y u^{-1 / 2}=O\left(Y u^{-1 / 2} t U^{-1}\right)=O\left(R U^{-1}\right) .
\end{aligned}
$$

Let $d^{\prime} \in(0, d)$ be arbitrary. By Lemma 2 we get

$$
\int_{d^{\prime}}^{d} e(u) F(u) d u=O\left(R U^{-1}\right) .
$$

As the implied constant does not depend on $d^{\prime}$, letting $d^{\prime} \rightarrow 0$ we get

$$
\int_{0}^{d} e(u) F(u) d u=O\left(R U^{-1}\right) .
$$

By Lemma 4(iv) we get

$$
\int_{d}^{\infty} e(u) u^{-1 / 2} d u=O\left(\min \left(1, d^{-1 / 2}\right)\right) .
$$

From (8) we deduce that $d=-A(c) \gg c^{2} / R$. As $c=\min (\varepsilon U, b-n)$ and $\left|f^{\prime}(b)-f^{\prime}(n)\right| \ll(b-n) / R$, and $n=n_{v}$ is such that $f^{\prime}(n)=v$, we get $d^{-1 / 2} \ll R^{1 / 2} c^{-1} \ll R^{1 / 2}\left(U^{-1}+(b-n)^{-1}\right) \ll R^{1 / 2}\left(U^{-1}+R^{-1}\left(v-f^{\prime}(b)\right)^{-1}\right)$, 
and thus

$$
\begin{aligned}
& \min \left(1, d^{-1 / 2}\right) \ll R^{1 / 2} U^{-1}+\min \left(1, R^{-1 / 2}\left(v-f^{\prime}(b)\right)^{-1}\right) \\
& Y \int_{d}^{\infty} e(u) u^{-1 / 2} d u=O\left(R^{1 / 2} \min \left(1, d^{-1 / 2}\right)\right) \\
&=O(R / U)+O\left(\min \left(R^{1 / 2}, \frac{1}{v-f^{\prime}(b)}\right)\right) .
\end{aligned}
$$

From Lemma 4(v), (5), (9) and (10), we get

$$
\text { (11) } \int_{0}^{c} e(-A(t)) d t=\frac{1}{4}(1+i) Y+O\left(R / U+\min \left(R^{1 / 2}, \frac{1}{v-f^{\prime}(b)}\right)\right) \text {. }
$$

As $(1-i) Y=-2 i\left|f^{\prime \prime}\left(n_{v}\right)\right|^{-1 / 2} e(1 / 8)$, from (3), (4) and (11), for $n_{v} \neq b$ we have

$$
\text { (12) } \begin{aligned}
\int_{n_{v}}^{b} e(f(x)-v x) d x= & -\frac{1}{2} i\left|f^{\prime \prime}\left(n_{v}\right)\right|^{-1 / 2} e\left(f\left(n_{v}\right)-v n_{v}+1 / 8\right) \\
& +O(R / U)+O\left(\min \left(R^{1 / 2}, \frac{1}{v-f^{\prime}(b)}\right)\right) .
\end{aligned}
$$

Similarly, for $n_{v} \neq a$ we can show that

$$
\begin{aligned}
\int_{a}^{n_{v}} e(f(x)-v x) d x= & -\frac{1}{2} i\left|f^{\prime \prime}\left(n_{v}\right)\right|^{-1 / 2} e\left(f\left(n_{v}\right)-v n_{v}+1 / 8\right) \\
& +O(R / U)+O\left(\min \left(R^{1 / 2}, \frac{1}{f^{\prime}(a)-v}\right)\right) .
\end{aligned}
$$

Thus, for $n_{v} \in(a, b)$ we have

$$
\begin{aligned}
& \int_{a}^{b} e(f(x)-v x) d x \\
& =-i\left|f^{\prime \prime}\left(n_{v}\right)\right|^{-1 / 2} e\left(f\left(n_{v}\right)-v n_{v}+1 / 8\right)+O(R / U) \\
& \quad+O\left(\min \left(R^{1 / 2}, \frac{1}{v-f^{\prime}(b)}\right)\right)+O\left(\min \left(R^{1 / 2}, \frac{1}{f^{\prime}(a)-v}\right)\right) .
\end{aligned}
$$

It is easy to observe that

$$
\begin{aligned}
& \text { (15) } \sum_{\alpha<v<\beta} \min \left(R^{1 / 2}, \frac{1}{v-\alpha}\right) \ll \min \left(R^{1 / 2}, 1 /\langle\alpha\rangle\right)+\log (\beta-\alpha+2), \\
& \text { (16) } \sum_{\alpha<v<\beta} \min \left(R^{1 / 2}, \frac{1}{\beta-v}\right) \ll \min \left(R^{1 / 2}, 1 /\langle\beta\rangle\right)+\log (\beta-\alpha+2),
\end{aligned}
$$


where $\alpha=f^{\prime}(b)<\beta=f^{\prime}(a)$. As $\beta-\alpha \ll R^{-1}(b-a)$, we have

$$
\begin{aligned}
& \log (\beta-\alpha+2) \ll \log \left(2+(b-a) R^{-1}\right), \\
& (\beta-\alpha+1) R U^{-1} \ll(b-a+R) U^{-1} .
\end{aligned}
$$

From (12) to (17) we get

$$
\begin{aligned}
& \sum_{\alpha \leq v \leq \beta} \int_{a}^{b} e(f(x)-v x) d x \\
& =-i \sum_{\alpha<v<\beta}\left|f^{\prime \prime}\left(x_{v}\right)\right|^{-1 / 2} e\left(f\left(x_{v}\right)-v x_{v}+1 / 8\right)+E, \\
& E=E_{1}+E_{2}+O\left(\log \left(2+(b-a) R^{-1}\right)\right)+O\left((b-a+R) U^{-1}\right) \\
& \quad+O\left(\min \left(R^{1 / 2}, \max (1 /\langle\alpha\rangle, 1 /\langle\beta\rangle)\right)\right) .
\end{aligned}
$$

If $v$ is an integer, $v \in(\alpha-1 / 2, \alpha)$, then $\alpha$ is not an integer, and we have

$$
f^{\prime}(x)-v \geq f^{\prime}(b)-v=\alpha-v=\|\alpha\|=\langle\alpha\rangle>0 .
$$

Thus by Lemmas 2 and 3 we get

$$
\sum_{v \in(\alpha-1 / 2, \alpha)} \int_{a}^{b} e(f(x)-v x) d x \ll \min \left(R^{1 / 2}, 1 /\langle\alpha\rangle\right),
$$

and this estimate also holds if $\alpha$ is an integer, in which case the interval $(\alpha-1 / 2, \alpha)$ does not contain integers. Similarly, we have

$$
\sum_{v \in(\beta, \beta+1 / 2)} \int_{a}^{b} e(f(x)-v x) d x \ll \min \left(R^{1 / 2}, 1 /\langle\beta\rangle\right) .
$$

Hence, from (2) and (18) we conclude that (1) holds with $\lambda=-i$. If $f^{\prime \prime}(x)>$ 0 for all $x \in[a, b]$, then by a similar argument, we can obtain (11) with $-A(t)$ and $v-f^{\prime}(b)$ being replaced by $A(t)$ and $f^{\prime}(b)-v$ respectively, and consequently, we should replace $-i$ in (12)-(14) and (18) by 1 , which results in the choice $\lambda=1$ in (1). This completes the proof of Theorem 1.

REMARK. In the proof of Theorem 1, we find that it is vital to evaluate the integral

$$
\int_{0}^{d} e(u) F(u) d u,
$$

which requires showing the monotonicity of the function $F(u)$. To this end, Min assumed that $f(x)$ is an algebraic function, from which he inferred that $F(u)$ is also an algebraic function, and thus is monotonic in $O(1)$ small intervals. The question of whether Min's argument is reasonable is beyond the range of this paper. (In Min's argument, $d$ is just $-A\left(b-n_{v}\right)$.) 
4. Proof of Theorem 2. Let $B$ and $\delta$ be two real numbers with $B \delta \neq 0$, $\delta \neq 1,2$. If $\delta \neq 1 / 2$, then

$$
\left|3 \beta_{4}(x)-5 \beta_{3}^{2}(x)\right|=x^{-2}|(\delta-2)(1-2 \delta)| \gg a^{-2} \quad \text { for } x \in[a, b] .
$$

Thus it is easy to see that the conditions of Theorem 1 are satisfied. Hence (1) holds. If $\delta=1 / 2$, then we need an alternative treatment. We recall that the condition $\left|3 \beta_{4}(x)-5 \beta_{3}^{2}(x)\right| \geq C_{6} a^{-2}$ is introduced to show the monotonicity of $F(u)$. In case $\delta=1 / 2$, suppose without loss of generality that $B>0$. Then

$$
\begin{aligned}
F(u)= & x_{v}^{1 / 2} B^{-1} F_{1}(\theta), \\
F_{1}(\theta)= & -2\left((1+\theta)^{-1 / 2}-1\right)^{-1} \\
& -\sqrt{2}\left(1+\frac{1}{2} \theta-(1+\theta)^{1 / 2}\right)^{-1 / 2}, \quad \theta=t(u) / x_{v} .
\end{aligned}
$$

As $1+\frac{1}{2} \theta-(1+\theta)^{1 / 2}=\frac{1}{2}\left((1+\theta)^{1 / 2}-1\right)^{2}, \theta>0$, it is easy to see that $F_{1}(u)=$ 2 , thus $F(u)$ keeps the value $2 x_{v}^{1 / 2} B^{-1}$ for $u \in[0, d]$, which completes the proof.

5. Proof of Theorem 3. Without loss of generality we may suppose that $\delta<1$. In this case $\alpha=f^{\prime}(b), \beta=f^{\prime}(a)$, and $\lambda=-i$. By Lemma 1 we get

$$
\begin{aligned}
\sum_{a \leq n \leq b} n^{-1 / 2} e(f(n))= & \sum_{\alpha-1 / 2<v<\beta+1 / 2} \int_{a}^{b} x^{-1 / 2} e(f(x)-v x) d x \\
& +O\left(a^{-1 / 2} \log (\beta-\alpha+2)\right) .
\end{aligned}
$$

Let $x_{v}$ be the solution of $f^{\prime}(x)=v$, where $\alpha \leq v \leq \beta$. Then $x_{v} \in[a, b]$. Assume that $x_{v} \neq b, \varepsilon$ is a sufficiently small given positive number depending on $\delta$, and $b^{\prime}=\min \left(b, x_{v}+\varepsilon a\right)$. For $b^{\prime}=x_{v}+\varepsilon a$, let

$$
G(x)=\frac{x^{-1 / 2}}{f^{\prime}(x)-v}, \quad b^{\prime} \leq x \leq b .
$$

We have

$$
G^{\prime}(x)=\frac{1}{2} B \delta\left((1-2 \delta) x^{\delta-1}+x_{v}^{\delta-1}\right)\left(f^{\prime}(x)-v\right)^{-2} .
$$

Thus $G^{\prime}(x)=0$ has at most one root for $x \in\left[b^{\prime}, b\right]$. Consequently, for a suitable number $b^{\prime \prime} \in\left[b^{\prime}, b\right], G(x)$ is monotonic in $\left[b^{\prime}, b^{\prime \prime}\right]$ and in $\left[b^{\prime \prime}, b\right]$. Let $R=\left(|B| a^{\delta-2}\right)^{-1}$. For $b^{\prime} \leq x \leq b$, as

$$
\left|f^{\prime}(x)-v\right|=\left|f^{\prime}(x)-f^{\prime}\left(x_{v}\right)\right| \gg R^{-1}\left(x-x_{v}\right) \gg a R^{-1},
$$

we have $G(x) \ll R a^{-3 / 2}$. Thus using Lemma 2 we get

$$
\int_{b^{\prime}}^{b} x^{-1 / 2} e(f(x)-v x) d x=O\left(R a^{-3 / 2}\right) .
$$


This estimate holds trivially for $b^{\prime}=b$. Let $c=b^{\prime}-x_{v}$. We get

$$
\int_{x_{v}}^{b^{\prime}} x^{-1 / 2} e(f(x)-v x) d x=e\left(f\left(x_{v}\right)-v x_{v}\right) \int_{0}^{c}\left(x_{v}+t\right)^{-1 / 2} e(A(t)) d t,
$$

where $A(t)=f\left(t+x_{v}\right)-f\left(x_{v}\right)-v t$. We have

$$
\begin{aligned}
& \int_{0}^{c}\left(x_{v}+t\right)^{-1 / 2} e(A(t)) d t \\
& \quad=x_{v}^{-1 / 2} \int_{0}^{c} e(A(t)) d t+\int_{0}^{c}\left(\left(x_{v}+t\right)^{-1 / 2}-x_{v}^{-1 / 2}\right) e(A(t)) d t .
\end{aligned}
$$

Let (we consider $H(0)$ as the limit of $H(t)$ as $t \rightarrow 0$ )

$$
H(t)=\left(\left(x_{v}+t\right)^{-1 / 2}-x_{v}^{-1 / 2}\right)\left(A^{\prime}(t)\right)^{-1}, \quad 0 \leq t \leq c .
$$

As $A^{\prime}(t)=f^{\prime}\left(t+x_{v}\right)-f^{\prime}\left(x_{v}\right)=\delta B\left(\left(t+x_{v}\right)^{\delta-1}-x_{v}^{\delta-1}\right)$, we have

$$
\begin{aligned}
H^{\prime}(t)= & \frac{\delta B\left(-\frac{1}{2}\left(x_{v}+t\right)^{-3 / 2}\left(\left(t+x_{v}\right)^{\delta-1}-x_{v}^{\delta-1}\right)\right)}{\left(A^{\prime}(t)\right)^{2}} \\
& -\frac{\delta B(\delta-1)\left(\left(x_{v}+t\right)^{-1 / 2}-x_{v}^{-1 / 2}\right)\left(t+x_{v}\right)^{\delta-2}}{\left(A^{\prime}(t)\right)^{2}} .
\end{aligned}
$$

By means of Taylor expansions we get

$$
H^{\prime}(t)=\delta B x_{v}^{-5 / 2}\left(\frac{1}{8}(\delta-1)(2 \delta-1)\left(t / x_{v}\right)^{2}+O\left(t^{3} x_{v}^{-3}\right)\right)\left(A^{\prime}(t)\right)^{-2} .
$$

Thus $H^{\prime}(t)$ does not change sign for $\delta \neq 1 / 2$, and so $H(t)$ is monotonic. In case $\delta=1 / 2$, we find that $H(t)=2 B^{-1}$. Hence $H(t)$ is always a monotonic function for $0 \leq t \leq c$. Also, by using the Taylor expansion we find that $H(t)=O\left(R a^{-3 / 2}\right)$. Thus by Lemma 2 we get

$$
\int_{0}^{c}\left(\left(x_{v}+t\right)^{-1 / 2}-x_{v}^{-1 / 2}\right) e(A(t)) d t=O\left(R a^{-3 / 2}\right) .
$$

As $x_{v}^{-1 / 2}\left|f^{\prime \prime}\left(x_{v}\right)\right|^{-1 / 2}=C v^{-1 / 2}$, from (11) and (22)-(25) we get

$$
\begin{aligned}
\int_{x_{v}}^{b} x^{-1 / 2} e(f(x)-v x) d x= & -\frac{1}{2} C_{i v^{-1 / 2}} e\left(f\left(x_{v}\right)-v x_{v}+1 / 8\right) \\
& +O\left(R a^{-3 / 2}+a^{-1 / 2} \min \left(R^{1 / 2}, \frac{1}{v-\alpha}\right)\right) .
\end{aligned}
$$

The argument is then similar to (13)-(18) of Section 3, and we get 


$$
\begin{aligned}
& \sum_{\alpha \leq v \leq \beta} \int_{a}^{b} x^{-1 / 2} e(f(x)-v x) d x \\
= & -C i \sum_{\alpha \leq v \leq \beta} v^{-1 / 2} e\left(f\left(x_{v}\right)-v x_{v}+1 / 8\right)+E, \\
E= & E_{1}+E_{2}+O\left(a^{-1 / 2} \log \left(2+(b-a) R^{-1}\right)\right)+O\left((b-a+R) a^{-3 / 2}\right) \\
& +O\left(a^{-1 / 2} \min \left(R^{1 / 2}, \max (1 /\langle\alpha\rangle, 1 /\langle\beta\rangle)\right)\right),
\end{aligned}
$$

where $E_{1}$ and $E_{2}$ are defined in the statement of Theorem 3. For an integer $v \in(\alpha-1 / 2, \alpha)$ or $v \in(\beta, \beta+1 / 2)$, by taking derivatives it is easy to show that the function $x^{-1 / 2}\left(f^{\prime}(x)-v\right)^{-1}$ is monotonic in the intervals $\left[a, a^{\prime}\right]$ and $\left[a^{\prime}, b\right]$ respectively for a suitable $a^{\prime} \in[a, b]$. Thus similarly to (19) and (20) we can deduce from Lemmas 2 and 3 that

$$
\begin{gathered}
\sum_{\alpha-1 / 2<v<\alpha} \int_{a}^{b} x^{-1 / 2} e(f(x)-v x) d x \ll a^{-1 / 2} \min \left(R^{1 / 2}, 1 /\langle\alpha\rangle\right), \\
\sum_{\beta<v<\beta+1 / 2} \int_{a}^{b} x^{-1 / 2} e(f(x)-v x) d x \ll a^{-1 / 2} \min \left(R^{1 / 2}, 1 /\langle\beta\rangle\right) .
\end{gathered}
$$

Theorem 3 follows in view of (21) and (26). The case of $\delta>1$ can dealt with similarly, and we can get (26) with $-i$ being replaced by 1 , and $\alpha=f^{\prime}(a)$, $\beta=f^{\prime}(b)$. The proof of Theorem 3 is finished.

Using the method of deriving Theorem 3 , we can also get a similar result for the exponential sum $\sum_{a<n \leq b} n^{\mu} e(f(n))$, where $\mu<0, \mu \neq-1 / 2$, and $f(n)$ takes the form as stated in Theorem 3.

Appendix. The author has found on pp. 85-92 of the Selected Works of I. M. Vinogradov (Springer, Berlin, 1985) that an argument similar to Min's was already developed as early as 1927. Vinogradov's result is worse than Min's, and needs more conditions imposed on parameters. In both Vinogradov's and Min's method, an "algebraic function" is defined by an algebraic equation in two real variables. The modern version of this concept is not so simple, cf. also pp. 90-101 of C. L. Siegel's work Topics in Complex Function Theory, Vol. 1, Wiley, 1969.

\section{References}

[1] L. V. Ahlfors, Complex Analysis, McGraw-Hill, 1966.

[2] R. C. Baker and G. Harman, Numbers with a large prime factor, Acta Arith. 73 (1995), 119-145. 


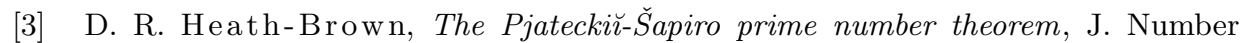
Theory 16 (1983), 242-266.

[4] C. H. Jia, The distribution of squarefree numbers, Sci. China Ser. A 36 (1993), 154-169.

[5] G. Kolesnik, On the order of $\zeta(1 / 2+i t)$ and $\Delta(R)$, Pacific J. Math. 98 (1982), $107-122$.

[6] H.-Q. Liu, On the number of abelian groups of a given order, Acta Arith. 59 (1991), 261-277.

[7] - On the number of abelian groups of a given order (supplement), ibid. 64 (1993), 285-296.

[8] - , The greatest prime factor of the integers in an interval, ibid. 65 (1993), 301-328.

[9] —, The number of cube-full numbers in an interval, ibid. 67 (1994), 1-12.

[10] - , The distribution of 4-full numbers, ibid. 67 (1994), 165-176.

[11] -, On some divisor problems, ibid. 68 (1994), 193-200.

[12] —, Divisor problems of 4 and 3 dimensions, ibid. 73 (1995), 249-269.

[13] - A special triple exponential sum, Mathematika 42 (1995), 137-143.

[14] —, Almost primes in short intervals, J. Number Theory 57 (1996), 301-322.

[15] H.-Q. Liu and J. Rivat, On the Pjateckir-Šapiro prime number theorem, Bull. London Math. Soc. 24 (1992), 143-147.

[16] S. H. Min, Methods in Number Theory, Vol. 2, Science Press, 1981 (in Chinese).

[17] E. Phillips, The zeta-function of Riemann, further developments of van der Corput's method, Quart. J. Math. 4 (1933), 209-225.

[18] E. C. Titchmarsh, The Theory of the Riemann Zeta-Function, 2nd ed., revised by D. R. Heath-Brown, Oxford, 1986.

[19] J. Wu, On the average number of unitary factors of finite abelian groups, Acta Arith. 84 (1998), 17-29.

[20] W.-G. Zhai and X.-D. Cao, On the average number of direct factors of finite abelian groups, ibid. 82 (1997), 45-55.

Department of Mathematics

Harbin Institute of Technology

Harbin, 150006, China
Address for correspondence: 206-10, Bao Guo Street Harbin, 150066, China

Received on 15.9.1998

and in revised form on 4.5 .1999 